\title{
N UEVOS EN FOQUES HISTÓRICOS E HISTORIA LITERARIA: HACIA LA CONSTRUCCIÓN DE UN MODELO CONCEPTUAL (EL CASO DEL CONCEPTO HISTORICISMO EN LAS HISTORIAS DE LA LITERATURA COLOMBIANA)*
}

\begin{abstract}
N EW HISTORICAL AN D LITERARY HISTORY PERSPECTIVES: TOWARDS THE CONSTRUCTION OF A CON CEPTUAL M ODEL (THE CASE OF THE CON CEPT HISTORICISM IN THE HISTORIES OF COLOMBIAN LITERATURE)
\end{abstract}

\section{GuStAVO AdolFo BEDOYA SÁN CHEZ**}

\section{RESUMEN}

Se busca señalar la importancia de los aportes conceptuales que la historia puede ofrecerle a la idea de una nueva historia de la literatura colombiana, ya que pensar en la escritura de una historia literaria significa reflexionar en torno a las diferencias conceptuales que dicha "nueva" historia literaria debe tener frente a la historia tradicional decimonónica (positivista, lineal, autoral, nacionalista), que aún hoy en día impera. Así, evidenciar las características de, por ejemplo: el positivismo, nos permitirá reflexionar sobre el papel que debe cumplir la historia literaria colombiana, para desasirse justamente de la forma de hacer historia clásica.

Palabras clave: Historia, literatura, positivismo, historia literaria colombiana, historiografía.

\section{ABSTRACT}

We signal the importance of the conceptual contributions that history can offer us for the idea of a new history of Colombian literature while thinking that the writing of a literary history means reflecting on the conceptual differences that the purportedly "new"

* El presente trabajo es resultado parcial del proyecto de investigación doctoral: "Nuevos enfoques históricos e historia literaria. Hacia la construcción de una historia socio-cultural de la novela colombiana (1914-2006)", que el autor adelanta en el Departamento de Historia de la Facultad de Ciencias Humanas y Económicas de la Universidad Nacional de Colombia (sede Medellín).

${ }^{*}$ Magíster en Literatura Colombiana de la Universidad de Antioquia (Medellín, Colombia). Docente de la Facultad de Comunicaciones de la Universidad de Antioquia y estudiante del Doctorado en Historia de la Universidad Nacional de Colombia (sede Medellín). E-mail: gustavoadolfo00@yahoo.com 
literary history should have when facing the traditional nineteenth century history (positivist, lineal, authorial, nationalist) that still reins today. And so, evidencing these characteristics (for example: positivism), will permit us to reflect on the role that Colombian literary history should play in order to separate ourselves from the form of writing of classical history.

Keywords: History, literature, positivism, Colombian literary history, historiography.

Recibido: 21.10.2008. Aprobado: 09.02.2009.

\section{PRESUPUESTOS: A MODO DE INTRODUCCIÓN}

Como disciplina de las ciencias sociales, la historia tiene una mayor tradición reflexionando en torno a los sustentos epistemológicos de la naturaleza de su objeto de ser, de allí por ejemplo, la existencia y fuerza de lo que comúnmente se conoce como filosofía de la historia. No sucede lo mismo con los estudios literarios, los cuales han tardado un poco más en configurarse como disciplina sistémica, y por ello su sustento epistemológico descansa en la existencia aislada de propuestas teóricas, críticas e históricas del hecho literario.

Así mismo, en la actualidad se reconoce la importancia (en el orden de la necesidad) de convocar al estudio en conjunto de diversos problemas de investigación a través del constante y activo diálogo de las distintas disciplinas de las ciencias sociales y humanas. Lo saben muy bien los interesados en la literatura y sobre todo aquellos que exigen de su estudio un acercamiento crítico, fundamentado conceptual y metodológicamente, tal como lo propusieron en su momento los formalistas rusos ${ }^{1}$. Pero lo saben sobremanera los interesados en la historia literaria que han empezado haciendo uso del llamado análisis historiográfico. Estos últimos convocan en sus discursos los esfuerzos y logros de otras disciplinas, tales como la historia, la sociología y la antropología, en aras de lo que en el mundo académico se ha querido designar bajo la perspectiva de saberes "trans", "multi" o pluridisciplinarios.

Decíamos arriba que lo sabían sobre todo los estudiosos de la historia literaria y la historiografía, y la razón se debe a que su disciplina de estudio, su parcela de actuación, parte del discurso histórico y no propiamente del literario. En otras palabras, su fundamentación epistemológica además de

\footnotetext{
${ }^{1}$ La intención del formalismo ruso, y posteriormente de aquellos que se ubican dentro de los estudios literarios, ha sido otorgarle a la investigación de la literatura unos principios y una metodología que le permita analizar, ordenar, clasificar y designar de forma precisa cada una de las singularidades de su materia.
} 
literaria es histórica. Por ello la necesidad de aunar fuerzas y compartir discusiones. Así, las presentes páginas buscan señalar la importancia de los aportes conceptuales que las tendencias históricas pueden ofrecerle a la idea de una nueva historia de la literatura colombiana, ya que pensar en la escritura de una historia literaria significa reflexionar en torno a las diferencias conceptuales que dicha "nueva" historia literaria debe tener frente a la historia tradicional decimonónica (positivista, lineal, autoral, nacionalista), que aún hoy en día impera.

De esta forma, y para el caso concreto de este artículo, evidenciar las características e implicaciones del uso conceptual del término "historicismo", nos permitirá reflexionar sobre el papel que debe cumplir la idea de escritura de una nueva historia de la literatura colombiana, pensada desde la actualidad.

\section{EL HISTORICISMO: SU CONDICIÓN DE POSIBILIDAD HISTÓRICA}

\section{Definición}

El historicismo admite diversas interpretaciones, por ello vemos la necesidad de matizar su utilización, siguiendo a Ramírez (2008: 430):

El historicismo puede contemplarse de diversos modos. La perspectiva epistemológica es uno de ellos, en cuanto permitió la institucionalización de la disciplina y ofreció herramientas metodológicas a otros humanistas. El término puede ser visto, de otro modo, como una perspectiva opuesta a la visión romántica o al naturalismo, que consideraba a la historia como creación de la naturaleza. El otro sentido es cuando se asimila como una visión del mundo teniendo en cuenta que la realidad humana puede comprenderse desde su desarrollo histórico. Esto implica una perspectiva de método, en cuanto que la historia puede ser vista como creación del hombre y, por lo tanto, se interesa por los hechos de los hombres, y está sujeta a lo singular e imprevisible de las motivaciones humanas.

El término apareció por primera vez a finales del siglo XIX designando las diversas maneras de pensar que enfatizaban la importancia del aspecto histórico del ser humano y de todo aquello que lo significa (costumbres, ideas, valores, etc.). Así, todos los fenómenos son producto de procesos que suceden en el tiempo, por lo tanto, la verdad estará condicionada de igual forma al paso de las generaciones: "El hombre es constitutivamente un ser a 


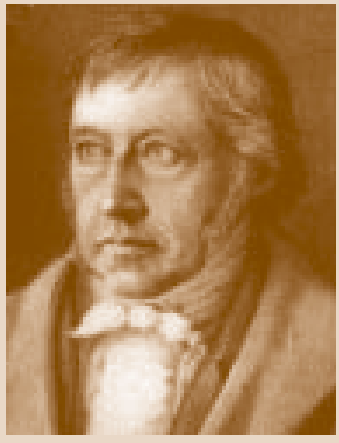

F. Hegel quien la historia siempre le concierne y no puede sino conocer las cosas desde la perspectiva concreta de su tiempo" (Cortés y Martínez, 1996).

\section{Antecedentes y consolidación}

La importancia concedida a la historia para explicar al hombre y a la sociedad puede ser rastreada en los sofistas griegos y en Giambattista Vico (16681744), pero adquirió relevancia con el desarrollo del nacionalismo. Así mismo, las discusiones en torno al dualismo "idealismo-racionalismo" hegeliano, el positivismo y la oposición ciencias naturales versus ciencias espirituales, permitieron el surgimiento y la consolidación del concepto. Examinemos cada una de estas condiciones:

El nacionalismo consideró y considera que la única posibilidad de llevar a cabo las aspiraciones sociales, económicas y culturales de un pueblo, es a través de la creación de un Estado-Nación. Las características que unen a dichas comunidades se derivan de la participación en común de ciertos lugares geográficos, de ciertas prácticas (por ejemplo, las religiosas) y de cierta lengua. La historia se constituyó en una de tantas maneras que el pueblo tuvo para dejar hablar a la patria, rescatando las particularidades de su devenir en el tiempo, exponiendo la importancia de su herencia cultural y justificando la identificación de la comunidad en la continuidad histórica.

Gracias al racionalismo hegeliano, el historicismo empezó a tener presencia en las reflexiones epistemológicas de la llamada filosofía clásica alemana. Basado en la fuerza y la capacidad de la razón para explicar el conocimiento, el racionalismo propone al hombre (al espíritu hegeliano) como sujeto de la historia. Georg Wilhelm Friedrich Hegel (1770-1831), en consonancia con el sentimiento nacionalista, argumenta que la historia culmina en la idea del Estado, a través de los ideales de razón y libertad (por supuesto, basado en su propuesta idealista del progreso). En otras palabras: estudiar la historia es estudiar la razón, ya que la historia es un proceso racional de la evolución de la libertad: la historia progresa desde una libertad menor hacia una libertad máxima (el mismo Estado).

El positivismo, como es bien sabido, se basa en la experiencia (empirismo) para explicar el conocimiento. Para ello defiende el papel de la descripción pura y establece la importancia de la historia como sinónimo de sistematización de las fuentes históricas. La historia, desde esta concepción, tiene un valor único, su principio: ordenar, su fin: el progreso. Auguste Comte (17981857), por ejemplo, rechaza toda concepción metafísica del pensamiento y por ello divide la historia en tres estadios según la fuente de su explicación: 
Estadio teológico (la explicación de los fenómenos proviene de la idea de Dios), Estadio metafísico (la explicación proviene de esencias abstractas o sobrenaturales) y Estadio positivo o científico (la explicación absoluta es rechazada, la atención debe centrarse en averiguar el cómo de los procesos, sus causas, con la intención de llegar a la postulación de generalizaciones sujetas, a su vez, a verificaciones comprobables).

Un último antecedente que debemos señalar como posibilidad de existencia del historicismo viene de la mano del filósofo Wilhelm Dilthey (18331911), quien en su división del mundo de las ciencias, en ciencias de la naturaleza y del espíritu, argumentó que el objeto de estas últimas es la realidad social: la base de las ciencias del espíritu es una "psicología", no explicativa sino descriptiva. Las ciencias del espíritu no estudian los fenómenos exteriores al hombre (y por lo tanto no los explican), sino que estudian las relaciones entre los individuos (su método es la comprensión). Todo ello le permite al autor presentar a la hermenéutica como técnica auxiliar de las ciencias del espíritu, acompañada del estudio histórico; sin esta perspectiva el conocimiento y el entendimiento sólo podrían ser parciales.

Estas iniciativas conceptuales lograron que durante el siglo XIX la historia se acreditara como disciplina científica, sobre todo en Francia y Alemania. Era tal su importancia que, por ejemplo, ingresó como cátedra en la Universidad de Berlín en el año 1910 (exactamente cien años después de que Wilhelm von Humboldt creara dicha universidad en el año 1810).

\section{El relativismo del historicismo}

La detracción más significativa que se le ha hecho al historicismo lo cuestiona por su carácter relativista, ya que si todo está condicionado por el tiempo histórico, no hay espacio para la verdad sustancial y el conocimiento humano. En últimas, el relativismo del historicismo tiende a ser más radical que el escepticismo, ya que mientras este último piensa que no hay verdades, o que son escasas, el relativismo argumenta que toda verdad tiene un valor relativo, dependiente de las condiciones históricas.

\section{Leopold von Ranke: el historiador}

Incorporándonos a la reflexión histórica, propiamente, y dejando atrás las discusiones innatas de la episteme del conocimiento, el nombre de Leopold von Ranke (1795-1886) es significativo. Considerado padre de la ciencia

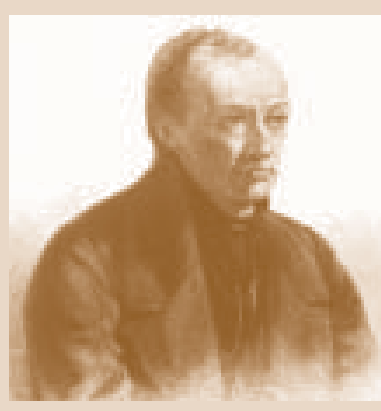

A. Comte

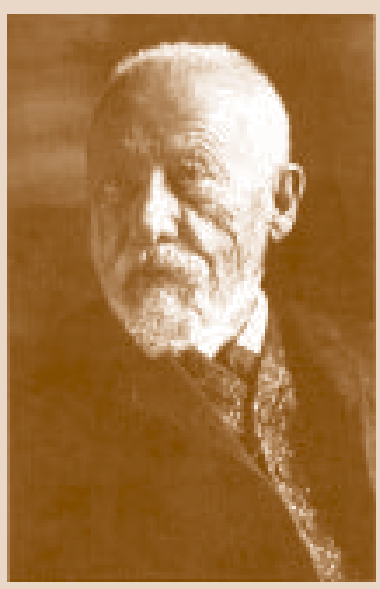

W. Dilthey 
histórica, se le debe la institucionalización de un método de enseñanza: el seminario deinvestigación histórica. Aunque su uso en las academias cobija a la más diversa variedad de disciplinas y especializaciones, el seminario de investigación o laboratorio de experimentación, pertenece en principio al estudio de la historia. La finalidad de estos espacios radicó en la presentación de los resultados parciales que conlleva la escritura de lo que en el futuro sería una tesis doctoral. Los seminarios de Ranke llevados a cabo en los años 1920 cobrarían con el tiempo cierto prestigio, sobre todo cuando empezaron a aparecer las primeras tesis, y con ellas, los primeros historiado$\mathrm{res}^{2}$. Los diversos seminarios ponen en práctica los principios que luego se constituirán en el legado del historicismo, a saber:

\section{La importancia de los documentos históricos}

Aunque siempre han existido personas dedicadas a la escritura de la historia, la historiografía anterior a Ranke había sido llevada a cabo por esfuerzos individuales, de forma netamente personal, guiadas por una pasión a retratar el pasado de su patria; así, la mayoría de estos trabajos estaba basado en la memoria, los recuerdos y las impresiones subjetivas, casi todos ellos escritos por aristócratas o diletantes testigos, que utilizaban la historia como método de preceptiva para educar la naciente juventud. Ranke reclamó la importancia y necesidad de basarse en fuentes históricas para entablar, en la medida de lo posible, un diálogo netamente objetivo de lo que se puede decir acerca del pasado.

\section{La crítica de fuentes}

Gracias al perfeccionamiento de una serie de técnicas se logró el análisis rigorista de los documentos y textos del pasado. Barthold Georg Niebuhr (1776-1831), por ejemplo, integró los diversos desarrollos de la gramática, la epigrafía, la filología, la paleografía, la numismática, la arqueología y la hermenéutica, para el estudio controlado, objetivo (aunque no incuestionable) de las fuentes históricas.

\footnotetext{
${ }^{2}$ Es significativo señalar la aparición de los textos de, entre otros, Ernst Bernheim (18501942), Charles-Victor Langlois (1863-1929) y Charles Seignobos (1854-1942) que intentaban dar cuenta de la nueva práctica de la escuela de historia alemana.
} 


\section{La delimitación de la ayuda imaginativa}

El historiador debe descubrir el contexto de su objeto de estudio para ofrecer un relato, en la medida de lo posible, verosímil, dejando a un lado su convicción o sus prejuicios.

Para Hayden White (1975: 163-164), los anteriores principios en Ranke se deben a:

Ranke had discovered that truth was stranger than fiction and infinitely more satisfying to him. He resolved, therefore, to limit himself in the future to the representation of only those facts that were attested but documentary evidence, to suppress the "Romantic" impulses in his own sentimental nature, and to write history in such a way as to relate only what had actually happened in the past. This repudiation of Romanticism was the basis of Ranke's brand of realistic historiography, a brand which, since Meinecke's popularization of the term, has come to be called "historism" and which still serves as the model of what an appropriately realistic and professionally responsible historiography ought to aspire to (163-164).

Paralelamente a la constitución de la historia como disciplina vital de las ciencias sociales, la práctica rankeana dará nacimiento a la historiografía, la cual se dedicó, de acuerdo con los postulados de su disciplina, a señalar plagios, dudas y manipulaciones de las fuentes documentales ${ }^{3}$.

\footnotetext{
${ }^{3}$ La concepción rankeana de la historia, correspondiente con sus posibilidades históricas, se concentra en la figura del Estado como conductor de la historia (sin olvidar a Dios como ser supremo), ya que es justamente el Estado-Nación el que está en un proceso de configuración, de desarrollo y progreso. Por todo ello su concepción historicista estará emparentada con la monarquía y no, por ejemplo, con las revoluciones burguesas del momento. En palabras de Hayden White (1975: 172): “In short, the 'idea of the nation' was for Ranke not only a datum but also a value; more, it was the principle in virtue of which everything in history could be assigned a positive or a negative significance. Ranke revealed as much when he characterized the idea of the nation' as eternal, changeless, a thought of God. He admitted that peoples may come and go, churches may for and disappear, and states may arise and perish; and that it is the historian's task to chronicle their passage or, in later times, to reconstruct them in their individuality and uniqueness". Más adelante: "Ranke regarded human problems as soluble only within the context of the nation and the institutions formed in the nation for those restraining impulses which he took to be inevitably destructive in their immediate forms of expression. He regarded anything that threatened the authority of the church (such as materialism and rationalism), of the state (such as capitalism, imperialism, racism, or Liberalism), or of the nation (such as Socialism, Communism, or ecumenical religion) as a threat to civilization itself" (175).
}

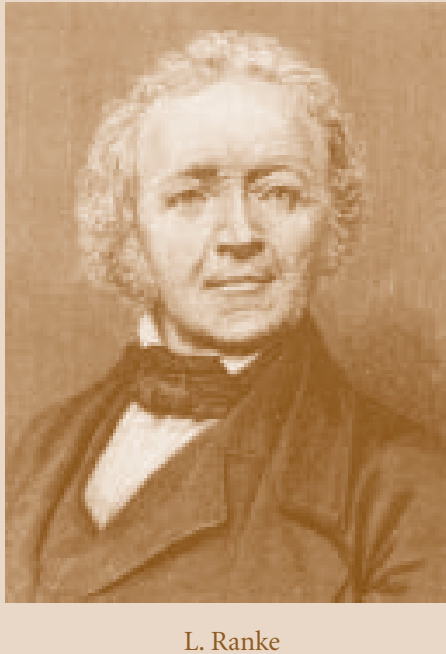

L. Ranke 
Podríamos decir que paralelo al éxito de los seminarios de investigación histórica de Ranke, nacieron los diversos mitos y descalificaciones de su concepción. La detracción más fuerte es aquella que asegura que la propuesta rankeana descansa en la "descripción" de los hechos tal como sucedieron, es decir, la tesis de que la historia no debe juzgar el pasado ni mucho menos instruir el tiempo presente:

A receptividade de Ranke nos Estados Unidos contribuiu para a idéia que freqüentemente se faz deste historiador como um empirista e fundador da história enquanto ciência. Mas este Ranke transformou-se em una espécie de antiquarista despreocupado com o sentido daquilo que estava descobrindo. A história escrita por ele ficou estigmatizada como uma negação da filosofia e do pensamento teórico (Guimarães Gaio, 2007: 90).

Sin embargo, salta a la vista que pensar el legado de Ranke desde la óptica empirista reduce la importancia del historiador y su perspectiva histórica. Su propuesta se basó, tal como dice Hayden White (1975: 164), en una crítica al romanticismo, un rechazo al filosofar a priori de Hegel (los principios de explicación mecanicista), en suma:

Ranke rejected anything that prevented the historian from seeing the historical field in its immediacy, its particularity, and its vividness.

This did not mean, as some of Ranke's interpreters have concluded, that his conception of objectivity approximated that of the naïve empiricist. Much more was involved in the world view which has since come to be called historism.

\section{CRÍTICA AL HISTORICISMO}

En la actualidad existe una mirada sesgada acerca del historicismo. En el mejor de los casos se le compara con la búsqueda bibliográfica y la búsqueda en archivos, y por ello no es extraño pensar en él como el cúmulo de "documentos históricos" (de pretendida autenticidad), organizados cronológicamente, sin utilidad aparente, pero donde descansa el "hecho histórico" a la espera de la visión profética del historiador ${ }^{4}$. Las críticas tomaron

\footnotetext{
${ }^{4}$ En algunos momentos se le ha comparado también con el antiquarianism, el cual Ritter (1986: 13-14) define como: "Interest in the past based on the love of old things. Learned curiosity about the past purely for its own sake, irrespective of present, future, or other broad interpretive
} 
diversos nombres, tales como "historia historizante" (Henri Berr) e "historia episódica” (événementielle: Paul Lacombe).

Se reprendió la naturaleza de los denominados hechos históricos del historicismo, ya que en la mayoría de los casos éstos respondían al carácter político, diplomático, militar y religioso de las sociedades.

También se ha reprochado acerca de la imparcialidad y objetividad del historiador, aunque, como lo han señalado los mismos historiadores, la descripción histórica no deja de ser (ni siquiera en el caso de la historia estructural) una narración.

Finalmente se le acusa de ser una disciplina fuertemente especializada y de objetar la ayuda de las teorías, es decir, de los planteamientos de cada uno de los conceptos utilizables en su discurso.

Las críticas son ciertas pero también lo es la explicación circunstancial del nacimiento de la historia en un momento en que, por ejemplo, la monarquía prusiana imperaba, así como una visión romántica y naturalista de la historia, es decir, la creencia de que los fenómenos sociales iban a darse en concordancia con los fenómenos naturales. En últimas, todos estos principios son condición de posibilidad, tanto de la misma disciplina como de sus problemas y objeciones posteriores.

\section{Escuela de los Annales}

A principios del siglo XX, en el seno de la llamada Escuela de los Annales, se originó una nueva serie de críticas al historicismo, justamente con la proclamación de la tildada Nueva Historia. Las propuestas, por ejemplo, de Fernand Braudel (1878-1956) y Ernst Labrousse (1895-1988) orientaron la investigación histórica de los acontecimientos hacia las estructuras. Así mismo, una serie de conceptos (no sólo de la Escuela de Annales sino de disciplinas hermanas, tales como la economía, el psicoanálisis, la antropología y la sociología, además del marxismo y el estructuralismo) aportaron nuevas discusiones y nuevas formas de comprender la disciplina histórica, conceptos tales como cuantificación, coyuntura, modelo, etc. En otras palabras, vino a darse lo que Peter Burke (1937-) llamó "una revolución historiográfica" (1999b), pues toda la concepción de la historia tradicional pasó a un segundo plano, y en el horizonte histórico empezaron a dominar una nueva

concerns; in the sense, normally pejorative. A branch of scholarship that flourished in Europe, particularly from the fifteenth to eighteenth centuries, devoted to the appreciation and classification of relics, monuments, and old texts". 


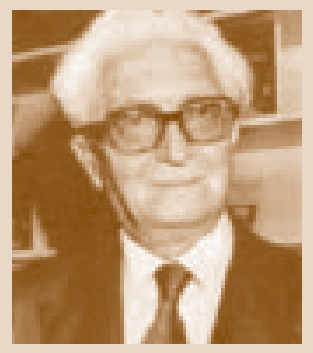

F. Braudel

serie de objetos de estudio, así como una nueva concepción metodológica y conceptual.

Del acontecimiento a la estructura, de la corta a la larga duración

Interesa sobremanera detenernos en la concepción de estructura de Fernand Braudel, que parece -a los ojos de la actualidad-constituirse en una de las mayores críticas de la historia de Annales al historicismo. Ante el riesgo del acontecimiento historicista, pero realmente ante el problema del tiempo en la historia (la duración), Braudel buscó, más allá del acontecimiento, las fluctuaciones del proceso coyuntural de duraciones diversas. De esta forma distinguió los tres niveles del tiempo histórico: tiempo corto (el nivel de los acontecimientos y de la historia episódica), el tiempo medio (el de las coyunturas, con ritmos más lentos pero variables) y el tiempo largo (el nivel profundo de la historia estructural, el tiempo más lento y de más larga duración). Escuchemos al propio Braudel (1979: xix):

Hemos llegado, así, a una descomposición de la historia por pisos. O, si se quiere, a la distinción, dentro del tiempo de la historia, de un tiempo geográfico, de un tiempo social y de un tiempo individual. O, si se prefiere esta otra fórmula, a la descomposición del hombre en un cortejo de personajes. Tal vez sea esto lo que menos se me perdonará; aunque afirme, defendiéndome de antemano, que también los recortes tradicionales fraccionan la historia viva y sustancialmente una; aunque sostenga, en contra de Ranke o de Karl Brandi, que la historia-relato no es un método, o no es el método objetivo por excelencia, sino también una filosofía de la historia: aunque asevere, y demuestre más adelante, que estos planos superpuestos no pretenden ser otra cosa que medios de exposición, y no me abstenga, ni mucho menos, de pasar de uno al otro sobre la marcha.

Así, fragmentando la realidad histórica en diversos niveles temporales de análisis, Braudel puso en tela de juicio la creencia de la historia decimonónica de que en cada periodo y sociedad los diferentes elementos transcurren simultáneamente, demostrando las diferencias de ritmo entre distintos sectores (lo económico, lo social, lo político, las mentalidades, lo geográfico, etc.).

\section{La miseria del historicismo}

Es necesario detenernos un momento en el libro de Karl Popper (1984) La miseria del historicismo, porque a partir de su título parece existir una crítica 
al concepto que nos ocupa. No es gratuito que la dedicatoria del libro sea: "En memoria de los incontables hombres y mujeres de todos los credos, naciones o razas que cayeron víctimas de la creencia fascista y comunista en las Leyes Inexorables del Destino Histórico".

En principio rescatemos una de las tantas definiciones que el autor hace del objeto de estudio: "Entiendo por historicismo un punto de vista sobre las ciencias sociales que supone que la predicción histórica es el fin principal de éstas, y que supone que este fin es alcanzable por medio del descubrimiento de los 'ritmos' o los 'modelos', de las 'leyes' o las 'tendencias' que yacen bajo la evolución de la historia" (17). Seguidamente, el autor diferencia a dicho "historicismo" en dos ramas: naturalistas y antinaturalistas, las cuales se diferencian en considerar o no, que algunos métodos de las ciencias naturales se pueden aplicar a las ciencias sociales.

En otras palabras, la propuesta del autor critica la creencia en el destino humano y la predicción de dicho destino por métodos racionales. En ningún momento cuestiona, por ejemplo, los principios del seminario de investigación histórica rankeana que dan origen a lo que hemos conocido desde el siglo XIX como historicismo. Según Cornblit (1996), el equívoco parece darse conceptualmente en el uso -por parte de Popper- del término "hystoricism", que no coincide con el de "historismus" de la filosofía de la historia, el cual es utilizado para designar la disciplina que ha pretendido entender la historia dentro de la evolución planteada por las ciencias naturales.

\section{EL HISTORICISMO Y LA HISTORIA DE LA LITERATURA COLOMBIANA}

En otras ocasiones (Bedoya, 2006a, 2006b) hemos dicho que algunos ejemplos de las llamadas "historias literarias", escritas en la Colombia del siglo XX y XXI, responden más a la visión decimonónica de análisis históricoliterario, es decir, a la propuesta historicista, que a los presupuestos interdisciplinarios de las actuales ciencias sociales y humanas. Sin embargo, hoy queremos ir un poco más lejos (y por ello, toda la contextualización que hemos hecho del historicismo), queremos asegurar que realmente el planteamiento de algunas propuestas de historia literaria del siglo XX y XXI colombiano, ni siquiera responden a esa visión (que, como hemos visto, fundamenta el estudio histórico e historiográfico), sino que se limitan a adaptar unos modelos preceptivos y de diletantismo, en la mayoría de los casos, 
que dejan a un lado los verdaderos intereses de la historia al modo clásico, tradicional, historicista.

Para ejemplificar el tipo de historia decimonónica (en parte historicista, pero de marcada tendencia positivista), haremos énfasis en la obra de José María Vergara y Vergara H istoria dela literatura dela N ueva Granada (1867). No sólo en su calidad de iniciadora de la tradición histórico-literaria nacional, sino como mayor influencia explícita, que se evidencia en la disciplina histórico-literaria -incluso-, hasta mediados y finales del siglo XX. Luego observaremos cómo su modelo es adaptado tan sólo en parte, y de allí que las historias "degeneren", en el mejor de los casos, en "manuales" de preceptiva. En otras palabras, decimos que la historia de la literatura colombiana más cercana al modelo historicista es la historia inicial, la historia de Vergara y Vergara, el resto de historias posteriores y que se dicen, partir o superar la propuesta de Vergara, sólo "mal-adaptan" la historia de Vergara.

\section{La historiografía literaria clásica colombiana. El estilo José M aría Vergara y Vergara}

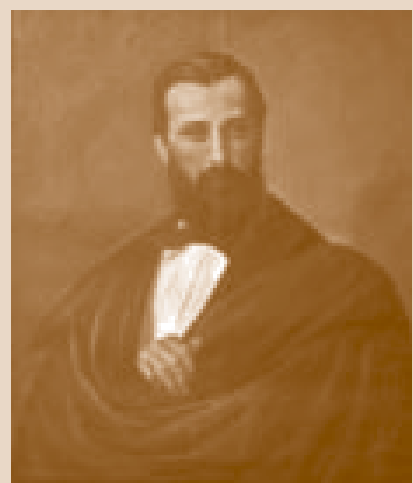

J.M. Vergara y Vergara
El historiador literario arriba señalado se propuso el análisis de la totalidad literaria nacional (todos los autores, las obras, los géneros y los fenómenos literarios) en el transcurso, también total, del tiempo (desde lo que el mismo historiador llama "inicios" de la literatura nacional hasta el momento de escritura de la historia). Vergara y Vergara publicó H istoria de la literatura dela Nueva Granada. Dela Conquista hasta la Independencia (1538-1820), y se planteó empezar, hasta que la muerte truncó el proyecto, $\mathrm{H}$ istoria dela literatura dela Nueva Granada. Cuadros políticoso díashistóricos (1849-1864).

Su obra cuenta con cinco ediciones, publicadas en fracciones de tiempo no mayor a 38 años y no menor a 18, desde 1905 hasta años recientes, para ser exactos, hasta el 2004. Cada nueva edición ha traído consigo una serie de adiciones y comentarios llevados a cabo por otros investigadores, diferentes al autor, lo que da clara muestra de la recepción de la obra ${ }^{5}$; recepción activa por parte de las demás historias literarias del siglo XIX y finales del XX, como lo demuestran los trabajos de Isidoro Laverde Amaya, Antonio Gómez Restrepo, Belisario Matos Hurtado, Jesús María Ruano, José Joaquín Ortega Torres, Nicolás Bayona Posada, Juan de Dios Arias, José A. Núñez Segura,

\footnotetext{
${ }^{5}$ Por ejemplo, la segunda edición (1905), además de un índice onomástico, cuenta con un prólogo, dos anexos y una serie de notas a pie de página escritas por los historiadores literarios Antonio Gómez Restrepo y Gustavo Otero Muñoz, en donde se contextualiza al lector sobre el
} 
Fernando Ayala Poveda, Yolanda Villamizar de Camperos, Francisco García Cárdenas y Félix Ramiro Lozada Flórez ${ }^{6}$. Dichos historiadores, en la mayoría de los casos, se declaran explícitamente deudores del trabajo de Vergara: "Rendir un homenaje de admiración y de respeto al iniciador de estos estudios en Colombia, al ilustre escritor don José María Vergara y Vergara, gloria de la literatura patria" (Gómez Restrepo, 1946: 7).

Por esto el servicio que el señor Vergara ha hecho a la historia literaria y a la historia política de nuestra patria, es inestimable; ha salvado, cuando estaban a punto de perecer, las reliquias del pensamiento de nuestros antepasados, que servirán a los futuros historiadores para explicar muchos sucesos preparatorios de los grandes acontecimientos de 1810, racionalmente inexplicables si no se conociera la tendencia de las ideas y la pujanza intelectual que, apenas instruidos, manifestaron los nativos de este suelo (Ancízar, 1943: 15).

En otros casos la evidencia de la práctica clásica, al estilo Vergara y Vergara, es apenas perceptible. Sin embargo, esta deuda, a pesar del tiempo y de los intentos de saldarla, representa un hecho curioso en la dinámica literaria nacional que demuestra la recepción plana, poco crítica, de aquella histo-

trabajo de Vergara y Vergara, se amplían algunos comentarios que el último dejó apenas planteados y se resaltan algunas incongruencias en el documento original. En la tercera edición (1931) se incluye la sección "Introducción-Reseña" escrita en 1870 por José Amador de los Ríos, en donde el historiador español analiza la importancia de la Historia de la literatura de la Nueva Granada, desde el cumplimiento de los objetivos de Vergara y Vergara. La cuarta edición (1956) viene acompañada con un prólogo biográfico escrito por Carlos Martínez Silva. La quinta edición (1974) se publica en dos pequeños tomos, conservando el contenido de la tercera edición. La sexta y más reciente edición (2004) presenta el contenido de la quinta y como novedad se da el hecho de que se publica con acceso libre en la Internet, en el link: http://www.lablaa.org/blaavirtual/literatura/ histolit/indicel.htm/

La relación y descripción de las historias de la literatura colombiana y de todos aquellos materiales idóneos a la hora de pensar una historia literaria se encuentran en: Vallejo, Olga y otros (2007). FEHLC, Fuentes para el estudio historiográfico de la literatura colombiana 1867-2007. Medellín: Universidad de Antioquia. Se trata de la recopilación crítica de más de 900 materiales histórico-literarios nacionales, de consulta libre en: http://ihlc.udea.edu.co/

${ }^{6}$ Como se puede observar, la influencia de Vergara y Vergara alcanza la obra de diversos historiadores que gracias a sus publicaciones y a las tantas ediciones y reediciones de éstas, se inscriben en el panorama histórico-literario del siglo XX y XXI.

Las presentes historias de la literatura representan un corpus mínimo de historias literarias con una clara perspectiva decimonónica, que han sido escritas -sin embargo-durante el siglo XX. Estas historias pueden organizarse, a su vez, en tres grupos:

Grupo 1. Historias que se declaran abiertamente deudoras de la tradición clásica: Arango (1950), Gómez (1946), Laverde (1882, 1963), Matos (1925), Núñez (1925), Ruano (1925).

Grupo 2. Historias que plantean un despegue de dicha propuesta aunque no lo logran: Ayala (1984), Lozada (2001).

Grupo 3. Historias recientes, finales del siglo XX, que a pesar de las propuestas teóricas, críticas e históricas literarias del presente, continúan perpetuando el modelo clásico: Rodríguez (2002), Villamizar (1997).

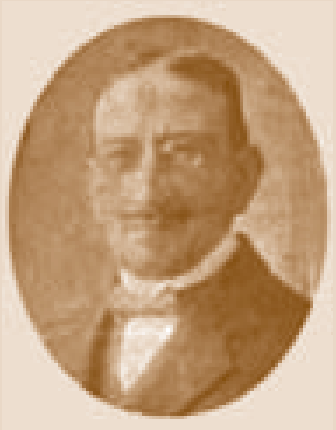

A. Gómez Restrepo

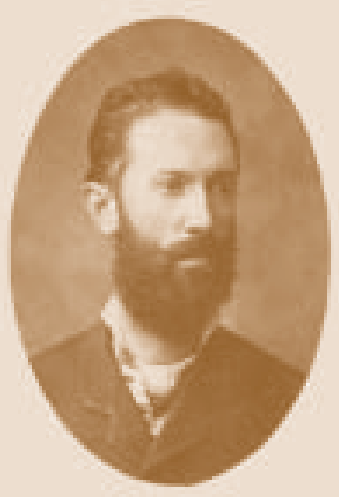

I. Laverde Amaya 
riografía clásica. Se trata de una forma de hacer historia a la antigua (de antes del historicismo), que ha imperado en el campo histórico-literario nacional durante todo el siglo XX, a pesar-como lo hemos venido repitiendo- del surgimiento de los estudios literarios, y de tantos otros discursos, desde la historia, pero también desde la sociología y la antropología, que han acompañado el devenir de las ciencias sociales y humanas:

Creemos que empujar a las ciencias sociales a combatir la fragmentación del conocimiento es empujarlas también en dirección a un grado significativo de objetividad. Creemos que insistir en que las ciencias sociales avancen hacia la inclusividad (en términos del reclutamiento de personal, la apertura a múltiples experiencias culturales, la lista de los temas de estudio legítimos) es tender a aumentar la posibilidad de un conocimiento más objetivo. Creemos que el énfasis en la historicidad de todos los fenómenos sociales tiende a reducir la tendencia a hacer abstracciones prematuras de la realidad y en definitiva ingenuas. Creemos que el cuestionamiento persistente en torno a los elementos subjetivos de nuestros modelos teóricos aumenta la probabilidad de que esos modelos sean relevantes y útiles (Wallerstein, 1996: 100).

Esta historiografía clásica, al estilo Vergara y Vergara, se caracteriza al responder de antemano a unas estructuras ideológicas de codificación del hecho literario. Dichas estructuras ideológicas pueden resumirse en tres tipos: políticas, religiosas e histórico-literarias. De esta forma la selección de las obras y los autores a incluirse en las historias, así como la forma en que se historian dichos elementos (es decir, el qué y el cómo historiar), responden a tales parámetros.

Políticamente la historiografía literaria clásica propugnó el modelo conservador, cuya meta fue generar una identidad nacional, pero -irónicamente- creada con base en la tradición hispánica. Escribir la historia literaria era (y sigue siendo) una acción patriótica y por ello se resaltan los escritores de literatura que también lo hacían, es decir, los escritores militantes, políticos; al igual que los próceres de la independencia y todos aquellos que lograron fundamentar la idea de independencia política y cultural: "La sinceridad me ha guiado, junto con el deseo de contribuir á (sic) popularizar los nombres de aquellos escritores que dan, con su pluma, honra y brillo á mi patria" (Laverde: 1882: iv). Así como Laverde, Matos Hurtado (1925: 3) escribió que su libro era "para honor de la República y gloria de la Literatura nacional" ${ }^{7}$. Por ello,

\footnotetext{
${ }^{7}$ Cuando la escritura de las historias pasa de manos de los conservadores a los liberales la idea sigue siendo la misma: demostrar la independencia política y cultural de la nación. La diferencia
} 
no es gratuito que el estudio de la literatura de Vergara y Vergara (2004: 219) culmine con la independencia política que equiparó a la soberanía intelectual y literaria, al mismo tiempo que advierte que: "El cultivo de la literatura francesa nos matará al fin. Debemos buscar por la literatura española el camino de la nuestra, hasta encontrar nuestra verdadera expresión nacional". La historiografía al estilo Vergara y Vergara atendió a los fundamentos católicos de la época. Así, era apenas lógico que llamara a su obra "un largo himno a la iglesia", puesto que "Cristiano, trabajo para mi religión; ciudadano, trabajo para mi patria" (24). Su intención era, al mismo tiempo, moralizadora y didáctica. Escribir literatura, así como escribir la historia literaria de la nación, corresponde a una actividad, además de patriótica, evangelizadora: no debe sorprender el hecho de que un buen número de historiadores en aquellos momentos fueran religiosos, al igual que los primeros autores que se resaltaban en las mismas historias, además de los próceres, fueran evangelizadores y cronistas que, según consideraciones de los historiadores, "cultivaron" el suelo de las letras nacionales para los escritores posteriores. Véase también, por ejemplo, la censura de Jesús María Ruano (1925), que marcó con un asterisco ${ }^{*}$ ), ante su contenido "poco moral", algunos títulos de José María Vargas Vila (1860-1933).

Como objetos dedicados al estudio histórico de la literatura, este tipo de historias expresan una visión positivista de la dinámica literaria y temporal en la que se inscriben los objetos literarios ficcionales. La dinámica literaria se basa en grandes figuras y acontecimientos, y no en los géneros, las mismas obras o los movimientos e influencias literarias. Esta sucesión de nombres y acontecimientos no da cuenta del devenir literario y mucho menos cultural o social de la nación. Se trata de una especie de evolucionismo entre los primeros autores y obras, y por supuesto, aquellas que representan más claramente el estatus ideológico del historiador. Para ser más claros, el propio Vergara y Vergara explicó su propósito: "seguir el orden cronológico, poniendo la noticia biográfica de cada autor y la de sus obras, y un breve juicio crítico sobre los escritos sobre el autor mismo; y mezclando todo esto con los sucesos referentes a las letras" (23).

Todo aquello que se escapaba a los parámetros ideológicos del historiador se desconocía en la historia literaria clásica, de allí su misma homogeneidad, homogeneidad que delimitaba claramente una tradición literaria culta e ilustrada; por ello la no inclusión en las primeras historias de cual-

radica en la separación de España y en la aceptación del pasado cultural amerindio por parte de los liberales.

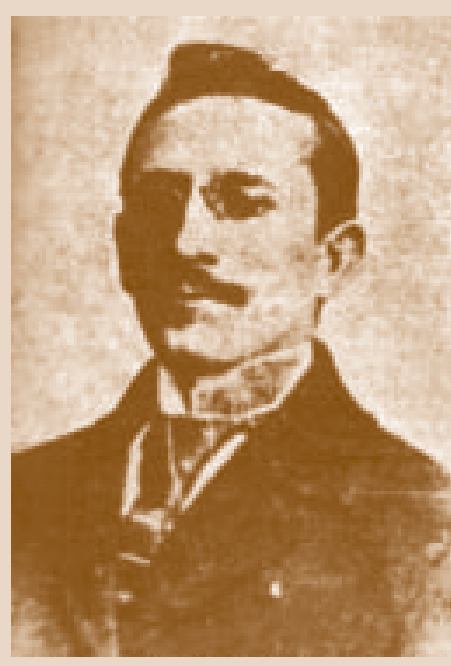

M. Vargas Vila 
quier manifestación de literatura popular, o la exclusión de autores que resaltaban el pasado indígena, tales como Próspero Pereira Gamba (1830-1896), Antonio Escobar (1879-1940) o Jesús Silvestre Rozo (1835-1895): "La historia de la literatura colombiana se presenta naturalmente dividida, á (sic) semejanza de la eclesiástica y civil, en dos épocas que caracterizan nuestra vida como nación: de la conquista á la independencia, y de la independencia para acá" (Laverde: 1882: 2). Las obras literarias eran valoradas en cuanto reafirmaran la propuesta ideológica del historiador, de esta propuesta es de lo que realmente da cuenta cada una de las historias literarias, y no, como lo supone el título historia literaria, del análisis en el tiempo, del hecho literario nacional.

\section{Las historias literarias posteriores: del acontecimiento a la anécdota}

Las condiciones de posibilidad de la historia literaria de Vergara y Vergara son claras: en un intento de Estado-Nación, de corte hispanista, conservador y creyente, la historia literaria inicial no podía ser otra (Véase: Gutiérrez Girardot, 1989). Sin embargo, las historias posteriores, que dicen partir de Vergara y Vergara para emularlo o superarlo, no pueden darse el mismo lujo de pensarse como historias inocentes que no tienen otra alternativa. En un momento en que los estudios literarios empezaban a configurarse como tal, y paralelos a los trabajos de críticos de la literatura colombiana (sin hablar de los adelantos en la disciplina histórica, pero también de la filosofía de la historia y la filosofía del pensamiento), algunos historiadores de la literatura plantearon un tipo de historia, menor en sus alcances, que el mismo tipo de historia propuesta por el historicismo decimonónico. En otras palabras: en el mismo momento que Annales criticaba e intentaba tomar distancia de la historia positivista e historicista, los historiadores de la literatura colombiana volvían al modo de historia pre-rankeano. Hacemos alusión al tipo de historia llevada a cabo por la famosa "autoridad", es decir, un gran nombre (en todos los casos masculino), destacado en el mundo político (incluso religioso), tipo Antonio Gómez Restrepo, José A. Núñez Segura y Jesús María Ruano. Incluso en la actualidad vivimos el efecto de la condición de "eminencia", en el caso Fernando Ayala Poveda (citado constantemente en los libros de texto), donde se entiende por literatura colombiana lo que su Manual plantea tan sólo porque aparece allí (todo ello es causa inmediata también de las diversas ediciones que ha coleccionado a lo largo del siglo XX).

Este tipo de historia basa su investigación en la anécdota y el comentario subjetivo; más que materiales para el estudio de la historia literaria parecen 
historias de vida y de lecturas. La verdad es que si lo son no nos debe preocupar. Lo preocupante y crítico del asunto es que dichos materiales se lean como historias o manuales para la enseñanza de la literatura.

En ellos, la periodización literaria degenera en taxonomía estática. El desarrollo literario se convierte en evolucionismo científico, por supuesto: progresivo, por ejemplo, la presentación de la realidad literaria en ciclos de tiempo, cada uno de ellos de alcances estilísticos mayores que el anterior, hasta dar fruto al hito literario, llámese Jorge Isaacs, José Eustasio Rivera, Gustavo Álvarez Gardeazábal o Gabriel García Márquez (por no señalar ciertas historias que predicen los nombres de autores tales como Héctor Abad Faciolince, o en el peor de los casos, Jorge Franco Ramos).

Lo mismo sucede con las corrientes y movimientos literarios (el paso del romanticismo al realismo y posteriormente al modernismo), e incluso en la clasificación genérica de la literatura (el cuento costumbrista se transforma en novela realista y luego en novela experimental, finalmente en novela del boom).

Cuando pensamos en estas historias no pensamos en los ideales historicistas: valoración de los documentos históricos, crítica de fuentes y delimitación de la ayuda imaginativa. Al contrario, las inexactitudes son frecuentes, por ejemplo, Ayala Poveda (en las primeras ediciones de su libro) habla de "las" novelas de Cepeda Samudio, cuando sólo escribió una. De igual forma, señala una fecha distinta de la real al nacimiento del autor.

Los estudios que otros historiadores hacen de la llamada "literatura colombiana" son parciales, por momentos dedican espacio a autores prácticamente desconocidos en el ámbito nacional, ¿ o cuántos estudiosos de la literatura colombiana saben que Félix Ramiro Lozada Flórez escribe poesía y por ello, su propia historia debe cerrarse con su nombre? Como podemos suponer, estas licencias eran imposibles en el laboratorio de investigación histórica rankeana.

\section{UNA NUEVA LECTURA DEL HISTORICISMO: A MODO DE CONCLUSIONES}

Ante la mirada sesgada que aún hoy en día tenemos acerca del historicismo, debemos recordar, por ejemplo, sus aportes: además del seminario de investigación histórica, la crítica de fuentes. Ambos permitieron el rechazo de la historia incipiente, la historia diletante, aficionada y entusiasta. Dichos aportes conllevan a su vez al necesario trabajo en los archivos; hoy en día, no sólo

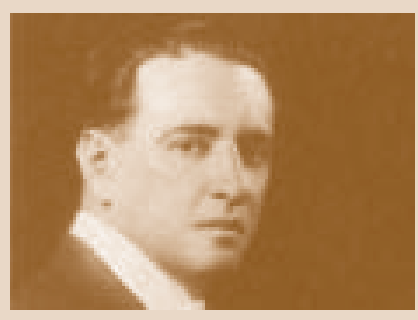

J.E. Rivera

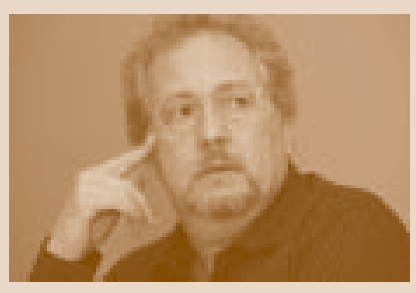

H. Abad Faciolince

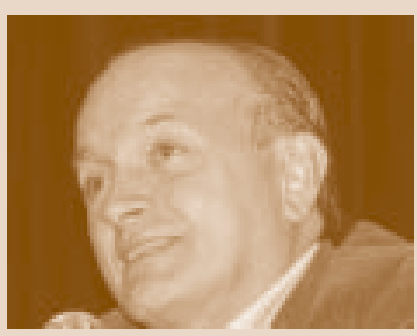

G. Álvarez Gardeazábal 
el archivo político, militar o religioso, sino cualquier tipo de archivo que el historiador sepa interrogar. La concepción de "verdad histórica", puesta en duda en la actualidad, logró -gracias al historicismo- dejar a un lado la ilación de sucesos. En conclusión, el historicismo tuvo una razón de ser en la historia para que ésta sea la disciplina que en la actualidad es, al mismo tiempo, el historicismo sigue siendo útil, pues estamos en total acuerdo con Cardoso y Pérez (1999: 20) cuando dicen: "Es necesario situar los documentos en el tiempo y el espacio, clasificarlos, criticarlos en cuanto a su autenticidad y credibilidad", y aunque dicho trabajo ya no representa la mayor parte de la actividad histórica, pensamos que aún hoy en día es necesario llamar la atención sobre dicho procedimiento, pues muchos -como hemos intentado mostrarlo- no lo hacen.

En definitiva, Ranke y el llamado historicismo dotaron a la historia de un método propio. Le abrieron paso al mundo universitario, y hoy más que nunca la máxima de no aceptar como válido algo que no sea comprobable (a partir de las evidencias documentales), es el principio motor de la historia como disciplina de confianza.

Mucho se ha dicho acerca de las inexactitudes del historicismo, se ha intentado pensar en soluciones o nuevos planteamiento que le den un giro a la problemática, por ejemplo a la historia-narración historicista se le ha dispuesto la antítesis historia-problema (Annales), sin embargo los mismos historiadores han confesado que la historia-problema, partiendo de la lectura que hace de cifras, cuadros estadísticos, en un intento de estudio netamente objetivo, sigue siendo narración. De esta forma, la lucha contra "la vieja" historia positivista se nutre actualmente del diálogo con otras ciencias sociales y humanas. En últimas, debe pensarse la realidad de que dichas limitaciones de la historia historicista no han sido saldadas, y su problemática permanece vigente.

Un último párrafo: si contemplamos el método rankeano, la historia debe enseñarse como sistema de investigación, como disposición de métodos y conceptos que permitan al estudiante descifrar el pasado y comprender las razones que explican su situación presente como sujeto histórico: una historia-herramienta, que alimente la capacidad crítica de los historiadores, la disciplina y la misma comprensión del mundo social.

\section{REFERENCIAS}

Ancízar, Manuel. 1943. "Prólogo", en: Vergara y Vergara (1958). Historia de la literatura de la N ueva Granada. Bogotá, Presidencia de la República. 
Arango H., Rubén. 1950. M i literatura. Crítica deliteratura colombiana. Medellín: Imprenta Departamental.

Ayala Poveda, Fernando. 1984. M anual de literatura colombiana. Bogotá: Educar Editores.

Bedoya, Gustavo. 2006a. "La periodización de la literatura colombiana en sus historias literarias: tradición y rupturas", en: Jalla 2006, Jornadas Andinas de Literatura Latinoamérica. Bogotá: Pensar. Cd-Rom.

2006b. "Problemas de la periodización literaria en las historias de la literatura colombiana: balance crítico”, en: Lingüística y Literatura. Medellín: Universidad de Antioquia, Vol. 27 No 49, pp. 95-114.

Braudel, Fernand. 1979. El mediterráneo y el mundo mediterráneo en la época de Felipell. México: Fondo de Cultura Económica.

Burke, Peter. 1999a. "Capítulo 1. Obertura: la nueva historia, su pasado y su futuro", en: Formas de hacer historia. Madrid: Alianza Editorial, pp. 11-37. . 1999b. La revolución historiográfica francesa. La escuela delosAnnales: 1929-1989. Barcelona: Gedisa.

Cardoso, Ciro y Pérez Brignoli, Héctor. 1999. Los métodos de la historia: Introducción a los problemas, métodos y técnicas de la historia demográfica, económica y social. Barcelona: Crítica.

Cornblit, Óscar. 1996. “Karl Popper, el historicismo y la narración”, en: Estudios Políticos. Santiago de Chile: Centro de Estudios Públicos, No 62, pp. 197-214.

Cortés Morató, Jordi y Martínez Riu, Antoni. 1996. Diccionario de filosofía en CD-ROM . Barcelona: Herder.

Gómez Restrepo, Antonio. 1946. Historia de la literatura colombiana. Bogotá: Imprenta Nacional.

Guimarães Gaio, Gessica Góes. 2007. "Ranke: o historicismo e a história científica”, en: A tarefa do H istoriador no alvorecer do H istoricismo. Río de Janeiro: Pontifícia Universidade Católica Do Rio de Janeiro, pp. 89-102.

Gutiérrez Girardot, Rafael. 1989. Temas y problemas de una historia social de la literatura hispanoamericana. Bogotá: Parsifal.

Laverde Amaya, Isidoro. 1882. A puntes sobre bibliografía colombiana con muestras escogidas en prosa y en verso. Santafé de Bogotá: Imprenta de Vapor de Zalamea Hermanos.

. 1963. Ojeada histórico-crítica sobre los orígenes de la literatura colombiana. Bogotá: Banco de la República, Talleres Gráficos.

Lozada Flórez, Félix Ramiro. 2001. Literatura colombiana. Neiva: Kimpres.

Matos Hurtado, Belisario. 1925. Compendio de la historia de la literatura colombiana para el uso de los colegios y de las escuelas superiores de la República. Bogotá: Ediciones Marconi.

Núñez Segura, José A., sj. 1925. Literatura colombiana (sinopsisy comentarios de autores representativos). Medellín: Editorial Bedout.

Popper, Karl. 1984. La miseria del historicismo. Madrid: Alianza Editorial.

Ramírez, Renzo. 2008. "El historicismo: profesionalización e ideologización de la historia”, en: Historia de las ideologías políticas: proyecto Ágora. Medellín: Fondo Editorial Universidad Eafit, Canal U, pp. 425-437. 
Ritter, Harry. 1986. Dictionary of concepts in history. London: Greenwood Publishing Group.

Rodríguez Ruiz, Jaime Alejandro. 2002. Novela colombiana. Bogotá: Pontificia Universidad Javeriana, Cd-Rom.

Ruano, Jesús María, sj. 1925. Resumen histórico-crítico dela literatura colombiana. Bogotá: Editorial Santafé.

Vallejo, Olga y otros. 2007. Fuentes para el estudio historiográfico de la literatura colombiana 1867-2007 FEH LC. Medellín: Universidad de Antioquia. Cd-Rom. Acceso libre en: http://ihlc.udea.edu.co/

Vergara y Vergara, José María. 2004. H istoria de la literatura dela N ueva Granada. Acceso libre en: http://www.lablaa.org/blaavirtual/literatura/histolit/ indicel.htm

Villamizar de Camperos, Yolanda. 1997. Literatura colombiana. Pamplona: Universidad de Pamplona, Centro de Estudios a Distancia, Programa de Español y Comunicación.

Wallerstein, Immanuel. 1996. Abrir las ciencias sociales. Informe de la Comisión Gulbenkian para la reestructuración delasciencias sociales. México: Siglo XXI Editores.

White, Hayden. 1975. M etahistory. The historical imagination in nineteenthcentury Europe. Baltimore y London: The Johns Hopkins University Press.

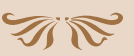

